\title{
1 Genomics-based annotations help unveil the molecular composition of edible plants
}

2 Ofaim Shany ${ }^{1}$, Menichetti Giulia ${ }^{1,2}$, Sebek Michael ${ }^{1}$ and Barabási Albert László ${ }^{1,2,3}$

$3{ }^{1}$ Network Science Institute and Department of Physics, Northeastern University, Boston, USA;

4 'Department of Medicine, Brigham and Women's Hospital, Harvard Medical School, Boston, USA;

$5 \quad{ }^{3}$ Department of Network and Data Science, Central European University, Budapest, Hungary.

7 Corresponding author

8 Correspondence to: a.barabasi@northeastern.edu

9

\section{Abstract}

Given the important role food plays in health and wellbeing, the past decades have seen considerable experimental efforts dedicated to mapping the chemical composition of food ingredients. As the composition of raw food is genetically predetermined, here we ask, to what degree can we rely on genomics to predict the chemical composition of natural ingredients. We therefore developed tools to unveil the chemical composition of 75 edible plants' genomes, finding that genome-based annotations increase the number of compounds linked to specific plants by 42 to $100 \%$. We rely on Gibbs free energy to identify compounds that accumulate in plants, i.e., those that are more likely to be detected experimentally. To quantify the accuracy of our predictions, we performed untargeted metabolomics on 13 plants, allowing us to experimentally confirm the detectability of the predicted compounds. For example, we find 59 novel compounds in corn, predicted by genomics annotations and supported by our experiments, 
23 but previously not assigned to the plant. Our study shows that genome-based annotations can

24 lead to an integrated metabologenomics platform capable of unveiling the chemical composition

25 of edible plants, and the biochemical pathways responsible for the observed compounds.

Background

"Make every bite count", recommends the U.S Departments of Agriculture (USDA)

Dietary guidelines for Americans $(2020-2025)^{1}$, reminding us of the multiple roles food, and

specifically fruits and vegetables, play in our wellbeing, serving as a source of energy and

nutrients, modulating our health, and affecting disease ${ }^{2-4}$. Plants are complex organisms antioxidant and anti-inflammatory activities effecting multiple diseases, from Cancer to diabetes or hypertension ${ }^{9}$.

42 USDA, despite the fact that the true number of chemical compounds in food ranges from tens ${ }^{10}$

43 to hundreds of thousands across all known plant species ${ }^{11}$. The bulk of our knowledge on the 44 chemical composition of food comes from mass spectrometry and other low-throughput 
analytical methods and is compiled in repositories such as FooDB ${ }^{12}$ and The Dictionary of Food

Compounds $^{13}(\mathrm{DFC})$, cataloguing comprehensive information on the detected compounds, including both evidence-based and predicted annotations. present in plants are encoded in the genome of the respective organism, encapsulating its metabolic capacities. Recent advancements in genomics have resulted in the emergence of extensive annotation efforts to decipher the genetic potential and the metabolic capacities of edible plants. For example, $\mathrm{KEGG}^{14}$ links genes to their functional annotations such as enzymes, reactions, and chemical compounds and catalogues them in metabolic pathway maps, offering

54 functional annotations for 7,254 organisms across the tree of life, out of which 56 are edible plants. Another contributor to plant genome metabolic annotations is PlantCyc ${ }^{15}$, a BioCyc ${ }^{16}$ based platform adapted to annotate the functional diversity of plant genomes. to expand the knowledge of the compound composition of foods. To do so we rely on metabologenomics ${ }^{17-19}$, to integrate genomics and metabolomics, used in the past to discover novel natural products ${ }^{17,20}$. To be specific, we develop a systematic metabologenomics pipeline, coupled with thermodynamic feasibility analysis aiming to predict the composition of edible plants. We validate our predictions by comparing them to the chemical knowledge curated by food composition databases like FooDB, DFC, and USDA4 . We also collect new experimental data

64 to explore the chemical composition of 13 plants. Our findings indicate that genomics-based 
composition of plant-based food, from fruits to vegetables and allow us to predict and experimentally test the presence of novel compounds in plants.

\section{Results}

\section{The existing knowledge on food composition}

We collected data for 75 edible plants with published and annotated genomes from two well established databases: KEGG and PlantCyc. Our collection represents plants from 28 families including monocots and dicots (Figure 1a), covering major plant food groups: fruits (apple, banana and orange), grains (rice, corn and quinoa), vegetables (tomato, potato and spinach) and proteins (soy, chickpeas and pigeon pea).

To estimate the currently available knowledge on the chemical composition of edible plants, we collected compound annotations from FooDB, DFC, and USDA, cataloguing 5,834, 5,151, and 140 compounds respectively, across all plants (Figure S1A). FooDB carries $723 \pm 452$ compounds on average per plant (median: 850 ), a number that can be as low as three compounds for clementine (citrus clementina) and as high as 2,181 compounds for tea (Camellia sinensis).

DFC carries $83 \pm 121$ compounds on average per plant (median:33) with as low as one compound for vegetable marrow (Cucurbita pepo subsp. pepo) and red rice (Oryza punctata) and as high as 697 compounds for tea. Finally, USDA carries $88 \pm 46$ compounds on average per plant (median:110), with a single compound for false flax (Camelina sativa) and Chinese white pear (Pyrus $\times$ bretschneideri) and 128 compounds for apple (Malus domestica). 
To estimate the contribution of genome-based annotations to the existing knowledge on food composition, we collected plant-related compounds from KEGG and PlantCyc. KEGG stores information about 7,245 organisms, out of which 546 are eukaryotes and 92 are plants, including 56 edible plants. PlantCyc is a plant-oriented database storing 126 genomes, out of which 58 are edible. These databases overlap and complement each other (39 plants overlap), together covering 75 metabolically annotated plant genomes. Overall, KEGG and PlantCyc contributed 1,201 and 3,737 new unique compounds respectively, adding a total of 5,224 new compounds (unique and common) to the composition of plants in our catalogue (Figure S1B). we focused on corn (Zea Mays), a highly consumed staple crop ${ }^{21}$ worldwide and in the US. Existing knowledge for corn includes 1,221 compounds from FooDB, 311 compounds from DFC and 127 compounds from USDA. Considering overlaps between all sources, this compiled to a total of 1,038 unique compounds. Next, we set out to explore the value of adding genome-based annotations to corn's existing knowledge. compounds, carried by a network of pathways. Some known pathways are only partially annotated even after considering multiple databases. For example, in Monoterpenoid biosynthesis in corn (Fig 2B) six out of nine compounds are annotated in both databases. Of the three remaining compounds, $(\mathrm{R})$-Ipsdienol is known to be present in food but was not annotated to any plant in our collection. The two remaining compounds, Ipsdienon, a product of the reaction catalyzed by EC 1.1.1.386 directly from (R)-Ipsdienol, and (6E)-8-Oxolinalool, a product 
110 (white circles, Figure 2b). To strengthen the stringency of our work, these compounds, whose

111 presence is documented in food, but not known to be associated with corn, are not included in

112 the plant's catalogue.

114 important class of compounds for health and nutrition ${ }^{22}$ ( $\alpha$-tocopherol better known as vitamin

E). Figure 2c shows the tocopherol biosynthesis pathway in corn and the delineated contribution

116 of each database to its compounds. We find that while databases such as FooDB and DFC

117 annotate the lower half of the pathway, capturing products such as vitamin $\mathrm{E}$ and its derivatives.

118 In contrast, genomics-based annotations offers a full pathway annotation, adding 4 new

119 intermediates: 3-(4-hydroxyphenyl)pyruvate, homogenistate, phytyl diphosphate and 2-methyl-

120 6-phytyl-1,4-benzoquinol and 2 new cofactors: S-adenosyl-L-homocysteine and S-adenosyl-L-

121 methionine, shedding light on the metabolic processes leading to the production of vitamin $\mathrm{E}$ in

122 corn.

Taken together, genomics-based annotations added 3,021 compounds to the list of

124 chemical compounds potentially present in corn, increasing its compound library by 64\% (Figure

125 2a). Across our collection, genomics-based annotations increased the number of compounds by

$1262,363 \pm 728$ chemicals on average per plant, an increase of $75 \% \pm 15 \%$. After this increase, our

127 database documents $3,239 \pm 1,054$ compounds per plant. We find that some plants are well

128 annotated in FooDB, DFC, and USDA, while others are poorly annotated (Figure 3). Tea (Camellia

129 sinensis) contains the highest number of FooDB, DFC, and USDA annotations (2,547 compounds

130 out of a total of 4,379$)$ showing an increase of $42 \%$ in new compounds. Some varieties of rice

131 (Oryza glaberrima, Oryza longistaminata, Oryza barthii), white yam (Dioscorea rotundata), wild 
132 tomato (Solanum pennellii) and woodland strawberry (Fragaria vesca) are not catalogued by

133 FooDB, DFC or USDA, hence for these plants genomics-based annotations contributed $100 \%$ of

134 the compounds. Other plants like clementine (citrus clementina), lotus (nelumbo nucifera) and

135 african oil palm (elaeis guineensis) are poorly annotated ( $<250$ compounds) in FooDB, DFC, and

136 USDA, hence the addition of genomics-based annotations increased our knowledge about their

137 chemical composition by more than $85 \%$.

\section{Pathway enrichment analysis}

Genomics-based annotations not only increase our knowledge about the chemical

composition of plants but also help us unveil the network of pathways responsible for the

142 production of the newly predicted chemical compounds. Indeed, metabolic pathway mapping

143 allows for a better understanding of the metabolic mechanisms responsible for the synthesis and

144 modulation of natural products and offer a knowledge base towards the prediction of currently

145 undetected compounds.

Metabolic pathways are divided into two main classes: primary and secondary

147 metabolism. Primary metabolism is the collection of pathways involved in growth, energy, and

148 reproduction of a plant, while secondary metabolism captures all other functions ${ }^{23}$, like flavonoid

149 biosynthesis, xenobiotics metabolism or plant-hormone metabolism to name only a few. For corn

150 we collected 789 pathways out of which $35 \%$ belong to primary and $65 \%$ belong to secondary

151 metabolism. We asked if certain pathways are represented more than others in our catalogue.

152 To test for pathway bias we performed a hypergeometric enrichment test capturing the chance

153 for a set of compounds mapped to a pathway in a certain plant to exceed the expected overlap 
with the general reference pathway ( $p$-value <0.05) (Figure S2). In corn, we found 479 enriched pathways spanning both primary (45\%) and secondary metabolism (55\%), indicating that our corn dataset is metabolically diverse.

We next asked about specialized metabolism occurring only in corn, scanning our plant collection for enriched pathways specific to it (Figure 4a). Corn-specific pathways include specialized metabolism like kauralexin and zealexin biosynthesis ${ }^{24,25}$, maysin biosynthesis ${ }^{26}$, bergamotene biosynthesis ${ }^{27}$ and all-trans-farnesol biosynthesis ${ }^{28}$. Each of these natural products of the diterpenoid, volatile sesquiterpenes and flavone families are produced by the plant to acquire resistance against biotic and abiotic stress, such as herbivore attack. primary/secondary in our entire collection. We identified 789 pathways across the full plant catalogue, $35 \%$ of which are related to primary and $65 \%$ to secondary metabolism, a fraction similar to the one observed for corn, offering evidence of diversity in our catalogue (Figure 4b,

167 e). We find most pathways to be enriched (p-value <0.05), with the exception of pathways belonging to glycan biosynthesis and secondary biosynthesis metabolism. Other pathways belonging to secondary metabolism showed a large variation in p-values as multiple outliers were observed above the enrichment line (Figure 4b).

We found 762 enriched pathways across all plants ( $p$-value $<0.05$ ), out of which $34 \%$ are

172 related to primary and $66 \%$ are related to secondary metabolism. The distribution of the number

173 of enriched pathways per plant shows two peaks (Figure 4c), corresponding to the two databases,

174 KEGG and PlantCyc. The peaks capture the fact that KEGG has fewer, more complex and generic 175 pathway map representations while PlantCyc has a larger number of smaller pathways. For 
example, KEGG represents the metabolism of alanine, aspartate and glutamate in one map while PlantCyc breaks down this process into 10 smaller pathways.

Different plants are known for their production of specialized compounds and natural products. Examples include the production of curcumin by turmeric, thymol by thyme and vanillin by vanilla plants ${ }^{29}$, prompting us to identify pathways enriched in single plants, pointing towards unique functionalities. Indeed, we observed a bimodal distribution of the number of plants per enriched pathway, one of the peaks being closer to 60 plants and another around 5 plants, indicating the presence of specialized plant metabolism in our catalogue (Figure 4c). Overall, we find that 30 out of 75 plants have at least one plant-specific enriched pathway (Figure S3) out of which we explored seven (Figure 4a). An example of such unique pathway is anthocyanin biosynthesis (delphinidin 3-O-glucoside) in grapes (Vitis vinifera), present in seeds and grape skins and used to differentiate between types of wine ${ }^{30,31}$. Other examples include: (1) hordatine biosynthesis in Barley (Hordeum vulgare subsp. vulgare). Hordatine, an antifungal compound, highly abundant in young barley shoots, reported to be a potential inhibitor of two main COVID19 proteins, a protease (PDB ID: 7BQY) and a RNA polymerase (PDB ID: 7bV2) ${ }^{32}$ and found in measurable quantities in different types of beer ${ }^{33}$. (2) Ricinoleate biosynthesis, found in castor bean (Ricinus communis) and the main constituent of castor oil, was shown to have antibacterial activities, and its elastic properties make it a candidate for packaging polymers with potential applications in biomedical and food technology ${ }^{34}$. (3) Capsaicin, a specialized component in pepper (Capsicum annuum) $)^{35}$ was recently shown to have positive dietetic effects and beneficial antioxidant activity through association with the gut microbiome ${ }^{36}$. (4) Sorgoleone, an allelochemical exuded from sorghum (Sorghum bicolor) roots is known to effect both microbial 
communities and neighboring plant growth ${ }^{37}$. Finally, we find a variety of acyl-sugar biosynthesis pathways in tomato (Solanum lycopersicum). Acyl-sugars are created in tomato trichomes and have commercial and medicinal $u^{3} \mathrm{~s}^{38}$. Another unique pathway in tomato is phenylpropanoid volatiles glycoconjugation, a pathway describing specialized volatiles found in tomato fruits contributing to its signature smell ${ }^{39}$.

In summary, genomics-based annotations bring diverse metabolic information from both primary and secondary metabolism, offering evidence of new specialized compounds.

\section{Experimental confirmation of genomics contribution to food knowledge}

To experimentally test the capacity of plant genomics to predict the presence of compounds in plants, we performed untargeted metabolomics experiments on 13 out of the 75 plants in our collection, resulting in a catalogue of 939 detected compounds (see Methods). On average, $371 \pm 130$ compounds were detected per plant, ranging from 264 in pear to 652 in apple, and 370 compounds for corn (39.4\% of our experimental catalogue). These experimental results allow us to evaluate the accuracy of genomics-based predictions. For this, we measured the overlap of the chemical structures between experimentally detected compounds and our known genomics-based compound collection, finding that genomics-based annotations show a significant overlap with experiments ( $p$-value $=0.018, \mathrm{SI}$ section 1 ).

To be specific, we identify 59 compounds that are found only in genomics-based annotations and were also detected in our experiments results (Figure 5a). We clustered these new compounds based on their chemical structure and classified them into primary and secondary metabolism (Figure 5b, Figure S5), finding that the majority of compounds were 
attributed to primary metabolism (30) and might represent pooled intermediates and their

221 possible fragments. Metabolite detection may also vary depending on the compound and spectra

222 libraries used in identification. As primary compounds are better studied and annotated,

223 secondary metabolites and their fragmentation products are less abundant in spectra libraries ${ }^{40}$.

224 Overall, we observed well characterized fractions of lipids, cofactors, sugar and amino acid

225 derivatives.

We identified seven compounds, present in corn that might potentially affect human well-

227 being (Figure 5c). For example, Citrulline, a non-essential amino acid known to effect

228 cardiovascular health and dilate blood vessels, and primarily found in watermelon and in smaller

229 amounts in other fruits including a variety of corn species ${ }^{41}$, is marketed as a dietary supplement

230 for bodybuilders and athletes to improve exercise endurance. N-Acetyl-D-Glucosamine,

231 previously detected in the shell that protects the first leaf of a corn shoot ${ }^{42}$, is known to help

232 support the joints and may help promote healthy skin ${ }^{43}$. Another compound detected is

233 nicotinamide ribonucleotide (NMN), reported to be detected together with NAD+, a well-known

234 cofactor, always present in the cell ${ }^{44}$. Well-being benefits related to this compounds stem from

235 its effects on NAD+ content. This compound has been well studied and as a member of the

236 vitamin B3 family ${ }^{45}$ and is being used for the treatment of a number of cardiovascular,

237 neurodegenerative and metabolic disorders ${ }^{46}$. Other compounds detected in corn and unveiled

238 by genomics-based annotations have potential cancer related effects. For example, 5-

239 methylthioadenosine (MTA), a sulfur-containing nucleoside has recently reported as tumor

240 suppresser $^{47}$. Another cancer associated compound, hypotaurine, a sulfinic acid with antioxidant

241 properties, derived from cysteine and an intermediate in taurine production, is one of the top 
242 ranked metabolites for differentiating low and high grade tumors ${ }^{48}$ but was also shown to evoke

243 a malignant phenotype in glioma, the most common primary brain malignancies in adults ${ }^{49}$. It is

244 also marketed as a dietary supplement, together with taurine and L-carnitine and associated with

245 semen quality improvement ${ }^{50}$. Some other compounds observed in this subset were suggested

246 to have both a well-being and economic/industrial importance. For example, Pipecolic acid, a

247 product of lysine metabolism, is an important regulator of immunity in plants and humans. In

248 plants, it accumulates upon pathogen infection and is associated with systemic acquired

249 resistance $(S A R)^{51}$. Pipecolic acid is also an important intermediate of pharmaceutically and

250 biologically derived compounds such as immunosuppressive agents and antibiotics ${ }^{52,53}$.

251 Our experimental investigation also unveiled 22 compounds found only in FooDB, DFC

252 and USDA. These include: (1) 6-Methoxy-2(3H)-benzoxazolone (MBOA), a degradation product

253 of the known bioactive compound 2,4-dihydroxy-7-methoxy-2H-1,4-benzoxazin-3(4H)-one

254 (DIMBOA). DIMBOA is the main benzoxazinone synthesized in young corn tissues and

255 accumulates in the cells. It is exuded by the roots and acts as a biocide against pests and as an

256 attractant for soil bacteria. MBOA, the more stable form, is often detected in corn soils ${ }^{54}$.

257 Interestingly, while DIMBOA is annotated to the corn genome, MBOA, its derivative is not. (2)

258 Vanillic acid, a phenolic detected in corn grits ${ }^{55}$, (3) Trigonelline, an active alkaloid known to be

259 found in corn and associated with antioxidant, anti-carcinogenic, anti-diabetic and anti-

260 hypercholesterolemia properties ${ }^{56}$, (4) Syringic acid, a phenolic compound found in fruits and

261 vegetables including corn and reported to have anti-oxidant, antimicrobial, anti-inflammatory

262 and antiendotoxic properties ${ }^{57}$, and (5) Feruloylputrescine, a polyamine monoconjugate

263 previously detected in corn kernels ${ }^{58}$. 
Finally, we considered all the plants in our catalogue for which we have experimentally

(49 \pm 15 and $26 \pm 14$ compounds respectively, Figure S6). In other words, our analysis shows that composition, helping us uncover potentially novel bioactive compounds.

\section{Genomics annotations contribute to the feasibility of compound accumulation}

277 Indeed, transient compounds may be harder to detect. To study the likelihood of a compound to

278 accumulate we used Gibbs free energy $(\Delta \mathrm{G})$ reaction values combined with a genome-scale

279 metabolic network topology. To establish thermodynamic feasibility, we determine the

280 probability that a compound accumulates given all the reactions it takes part in the network context (Figure 6a). In other words, thermodynamic feasibility offers a method for compound

282 ranking based on cumulative $\Delta \mathrm{G}$ values. We collected $\Delta \mathrm{G}$ values from modelSEED ${ }^{59}$ and PlantCyc

283 and calculated the cumulative $\Delta G$ value (score) for each compound. If the compound acted as a 
positive value. Compounds with positive scores are produced more than consumed, thus they are likely to accumulate. The reaction representing the largest $\Delta G$ value is called a sink reaction, as it shifts the balance largely towards either the consumption or production of a compound. In corn, we scored 2,985 compounds (63\% of total compounds), out of which 1,460 have positive $\Delta G$ values, i.e., are expected to accumulate.

To illustrate our findings, we explored the compounds on the vitamin E biosynthesis pathway in corn (Figure 6b). We observed likely accumulation of pathway products $\alpha$ and $\beta$ tocopherol and of the intermediates $\gamma$ and $\delta$ tocopherol. Intermediates shown to accumulate are likely involved in more than one reaction outside the pathway. For example, phytyl diphosphate is involved in 7 reactions and 5 pathways. The largest $\Delta G$ value measured for this set of reactions representing the sink was annotated to the phytyl salvage pathway, describing the conversion of degraded chlorophyl to phytyl phosphate, contributing to its high likelyhood to accumulate. A compound could likely accumulate if it is an intermediate appearing in two reactions with a large $\Delta G$ value difference. For example, 2,3-dimethyl-6-phytyl-1,4-benzoquinol is involved in two reactions, both annotated to the vitamin $E$ biosynthesis pathway, where the $\Delta G$ value for the reaction producing it $(20.64 \mathrm{kcal} / \mathrm{mol})$ is larger than the $\Delta \mathrm{G}$ value for the reaction consuming it ($12.05 \mathrm{kcal} / \mathrm{mol})$. Overall, $1,754 \pm 588$ compounds were scored per plant, covering an average of $79 \pm 17 \%$ of its compounds. The fraction of scored compounds was as high as $96.5 \%$ of the total compounds in Oryza longstaminata, a species of rice, and as low as $50 \%$ in adzuki bean (Vigna angularis).

To estimate the predictive power of our approach, we first asked if kinetics-based annotations have increased the predictive power of our platform compared to total genomics- 
based annotations. We find that Kinetics-based annotations show a more significant overlap with the experiments compared to genomics ( $p$-value=0.0018, SI section 1, Figure S4), and they are characterized by a high degree of structural similarity, significantly different from a random sample of the same size from genomics annotations ( $p$-value $<0.001$, SI section 1 ).

Next, we used the experimentally detected compounds in the 13 plants for which we performed untargeted metabolomics, to estimate the performance of our approach. Similar to known machine learning methods, we use $\Delta G$ scores as a 'classifier' predicting the likelihood of a compound to accumulate. We then compare it to our experimentally detected compound catalogue as ground truth values (a binary classification denoting presence or absence). We calculated standard performance metrics, such as the true positive and false positive rates and the area under the receiver-operator curve (ROC), $A \cup C_{R O C}$. In addition, we calculated the precision, recall and F-1 scores. Since our data may be imbalanced, we initially set the threshold of prediction to be larger than zero (positive values). We then performed a moving threshold analysis (see Methods) to determine the optimal threshold for best performance in each plant found in both our annotation and experimental catalogue (Table S1). Most $A U C_{R O C}$ values were above the discrimination line $(0.5)$, several representing acceptable discrimination $\left(A U C_{R O C}\right.$ values between 0.69 to 0.76 ) (Figure $6 \mathrm{c}$ ). Overall, $A \cup C_{R O C}$ values were better for the compounds that are expected to accumulate than the compounds predicted based on the whole genome, confirming the predictive power of this thermodynamics-based analysis.

Finally, we selected the top ranked 110 corn compound families with the best performance of our thermodynamics-based approach $\left(A \cup C_{R O C}=0.74\right)$. This list contained 15 experimentally detected compounds including AMP, S-adenosylhomocysteine (SAH) and 5- 
methylthioadenosine (MTA), which are cofactors maintained as constant pools in the cell. Other

products of major energy producing pathways, such as carbon fixation by photosynthesis,

glycolysis and the TCA cycle. Interestingly, L-glutamate and D-Galacturonic acid were also

detected. L-glutamate was previously reported as a key metabolite in corn, measured in the large

amounts in the endosperm ${ }^{60,61}$. D-Galacturonic acid is the main component of pectin, a

336 polysaccharide naturally found in plant cell walls. Thus, both compounds are likely to accumulate

337 and be detected in metabolomics measurements, supporting the predictive power of our 338 approach.

\section{Discussion}

Here we developed a systematic methodology to extract the contribution of genomicsannotations not only boosted, in some cases by more than $85 \%$, the number of compounds chemical composition of edible plants, specifically corn. 
352 signals, all governed by complex genetic and metabolic regulation. We therefore set out to 353 explore the feasibility of a compound to accumulate, affecting the likelihood of being 354 experimentally detectable. We find good discriminative power, supporting the large-scale use of 355 thermodynamic annotations. As experimental data and thermodynamics annotations expand, they may lead to significant enhancement in the predictive power of metabologenomics. While the advent of genomics offers new insights into the composition of edible plants, it

358 is not without limitations. Various biases that might arise from such data were explored 359 throughout this work, representing only a few of the multiple factors that might affect our 360 knowledge of food composition. One major limitation is the availability of annotated plant 361 genomes. While the cost of sequencing dropped significantly, we find that the number of non362 model plant genomes annotated remains limited and grows slowly. Other factors such as annotation quality and database standardization can also limit omics-based analysis. As shown here, two major databases, KEGG and PlantCyc, has introduced some redundancy in pathway mappings, partially rooted in the different pathway definitions used. As data continues to accumulate, standardization and mappings between the different data sources is key to deriving

367 new insights. Finally, the use of metabolomics to detect compounds in edible plants highly 368 depends on the instrumentation used, standard libraries and identification methods. For example, it is known that mass-spectrometers have poor detection of stereoisomers ${ }^{62-64}$. Even 370 though considerable efforts have been made to establish spectral analysis pipelines, high371 throughput metabolomics compound identification remains limited. Future improvements in 372 metabolomics analysis and annotation could significantly enhance our knowledge of specialized 373 metabolites and natural products in edible plants. 
Finally, the genomics-based annotation analyzed here greatly contribute to our existing

375 knowledge of the composition of edible plants. The integration of genomics and metabolomics

376 has been suggested as a promising combination towards the and identification of promising

377 compounds ${ }^{65}$. Thus, this work offers a steppingstone towards a better understanding of food

378 composition, offering insights based on fast-growing computational and experimental datasets.

Methods

\section{Data collection}

Compound annotations were collected from open-source (FooDB and USDA) and

All annotations were completed with SMILES, InChIKey and mass. Plant diversity was analyzed

InChi keys representing chemical families. The database files are available on

\section{Mass spectrometry experiments}

A selection of 13 produce items were purchased from two local grocery stores (Whole

394 Foods Market and Stop \& Shop): apple, banana, basil, black bean, carrot, chickpea, corn, garlic, 395 lettuce, olive, onion, peach, pear, pepper, potato, spinach, soybean, strawberry, sugar beet, and 
tomato. Each produce item sample contained the combined material of six units (for example 6 apples) and was prepared in a humidity-controlled room with minimum light exposure. Sample preparation included peeling, chopping, freeze drying $\left(-80^{\circ} \mathrm{C}\right.$ for $24 \mathrm{hrs}$, Catalog No. $10-269-56 \mathrm{~B}$ from LabConco/Fisher) and pulverizing into a fine homogenized powder (Kitchen Aid, 170W, Model No. BCG1110BO). All samples were prepared by the Giese lab (Northeastern University, Boston MA USA). The final samples were stored at $-80^{\circ} \mathrm{C}$ in vials containing $200 \mathrm{mg}$ of powder 402 and argon gas. The samples were shipped to two metabolomics centers (West Coast Metabolomics Center, UC Davis, CA USA and Metabolon, Morrisville, NC USA) for analysis on multiple platforms including UHPLC-CSH C18-HRMS-Orbitrap, UHPLC-BEH Amide-HRMSOrbitrap, UHPLC-PFP-HRMS-Orbitrap, and Shimadzu LC and SelexION QTRAP MS and annotation 406 (see SI section 2). standard library. Results from both metabolomics centers were merged and standardized to InChIKeys (PubChem). Since metabolomics methodologies does not account for stereochemistry, only the first block of the InChIKey is used to compare two entries. Therefore, the resulting list is one of unique compound structures found in each food item.

\section{Statistics and enrichment tests}


418

419

420

421

422

423

424

425

426

427

428

429

430

431

432

433

434

435

436

437 compounds found in each pathway in the organism and tested it for significance against the reference. To account for multiple testing on the same pathway, Bonferroni correction was applied to pathways' p-values.

\section{Compound similarity across genomics, kinetics, and experiments}

To investigate the degree of structural similarity and overlap between molecules retrieved by different techniques, we performed similarity search and clustering on a variety of molecular fingerprints (FP). We leveraged the python package RDKit ${ }^{68}$ to standardize SMILES and InChIKeys associated with each chemical annotation, using Morgan fingerprints (FP). To generate a Morgan FP, all substructures around the heavy atoms of a molecule within a defined radius are generated and assigned to unique identifiers. These identifiers are then hashed to a vector with a fixed length. The chemoinformatic community employs 1024- or 2048-bit vectors, populating them with fragments up to radius 1 or 2 . For our analysis we increased the resolution up to 8192 bits and radius 3 , to capture fragments of bigger size and reduce the potential bit collision ${ }^{69}$.

Since the first block of the InChIKey represents several stereoisomers, we assign a bit vector to each first block as the union of all bit vectors representing the related isomers. We then compare the degree of structural similarity between any pair of compounds by computing the Jaccard similarity between binary vectors.

We assess structural similarity for a given set of $N$ chemical compounds, by calculating the intrinsic dimension of their Jaccard similarity matrix $\left\{S_{i j}\right\}$, a function of the spectrum $\left\{\lambda_{i}\right\}$ of $\left\{S_{i j}\right\}$,

$$
n=e^{-\sum_{i=1}^{N} \frac{\lambda_{i}}{\sum_{k=1}^{N} \lambda_{k}} \log \left(\frac{\lambda_{i}}{\sum_{k=1}^{N} \lambda_{k}}\right)}=e^{H}
$$


439 where the Shannon entropy $H$ of the normalized spectrum is used to estimate the number of

440 independent components synthetizing the same amount of structural similarity observed in the

441 sample. The higher is $n$ the more chemically diverse is the sample. We used $n$ to assess how

442 different the kinetics annotations are compared to a random sample from the genomics set

443 (comparison with 1000 subsamples) (SI Section 1).

445 Thermodynamic feasibility analysis

We collected available thermodynamic annotations for all the plants in our collection.

447 Thermodynamic annotations in the form of $\Delta G$ values (Kcal/mol) were collected from $448 \quad$ ModelSEED ${ }^{59}$ and PlantCyc ${ }^{15}$.

As our validation set is based on metabolomics identified compounds, we used the first

451 information, a known limitation of mass spectrometry. To calculate the cumulative score of $\Delta \mathrm{Gs}$

452 for a compound we collected all the reaction annotations in which it is involved. If the compound

453 was acting as reactant in a reaction its $\Delta G$ value would be assigned a negative value and if it would

454 be a product, a positive value. All the values were then summed to represent a likelihood to

455 accumulate score where a positive value indicated a likely to accumulate compound and a

456 negative value a likely to be consumed value. This approach was applied for each plant in our

457 plant collection with corresponding experimental results.

458

459 Performance evaluation 
471 and updated performance metrics accordingly. All calculations were performed and plotted using the sklearn (version 0.24.1) and seaborn (version 0.11.1) python packages and Python 3.8).

\section{Acknowledgements}

475 This publication has received funding from the Rockefeller Foundation's 2109 FOD 026 grant.

476 Experiments performed at the West Coast Metabolomics Center at University of California, Davis

477 were led by Dr. Oliver Fiehn and Dr. Arpana Vaniya. Experiments at Metabolon were led by Nino 478 Esile, Dr. Brian Ingram, and Nathan Testa. We thank Dr. John de la Parra, and Rebekah Carlson

479 for preparing the food samples analyzed by UC Davis. We thank Dr. Roger Giese and Dr. Pushkar 


\section{Author contributions}

484 S.O performed the analysis and wrote the manuscript. G.M performed structural analysis, 485 structural enrichment, developed the intrinsic dimensionality approach to characterize the 486 structural redundancy of the Jaccard similarity matrix, and contributed to writing the manuscript.

487 M.S collected and analyzed the metabolomics experimental results. A.L.B conceived the project, 488 supervised, and contributed to the final version of this manuscript. All authors discussed the 489 results and contributed to the final manuscript.

\section{Competing interests}

493 A.L.B. is a scientific founder of Scipher Medicine, Inc., which applies network medicine strategies 494 to personalized drug selection, Foodome, Inc., which applies data science to food and health, and 495 Datapolis, Inc., which focuses on human mobility.

496

\section{Data availability}

498 The datasets generated during and/or analyzed during the current study are available on 499 https://github.com/Barabasi-Lab/Plant-genomics

\section{Code availability}

502 Code and scripts are available on https://github.com/Barabasi-Lab/Plant-genomics

\section{References}


1. Dietary Guidelines for Americans | USDA-FNS.

2. Luo, Y., Shang, P. \& Li, D. Luteolin: A Flavonoid that has multiple cardio-protective effects and its molecular mechanisms. Front. Pharmacol. 8, 1-10 (2017).

3. Barabási, A.-L., Menichetti, G. \& Loscalzo, J. The unmapped chemical complexity of our diet. Nat. Food 1, 33-37 (2020).

509

510

4. Hooton, F., Menichetti, G. \& Barabási, A.-L. Exploring food contents in scientific literature with FoodMine. Sci. Rep. 10, 16191 (2020).

5. Wimalanathan, K. \& Lawrence-Dill, C. J. Gene Ontology Meta Annotator for Plants (GOMAP). Plant Methods 2021171 17, 1-14 (2021).

6. Choi, I. Y., Kwon, E. C. \& Kim, N. S. The C- and G-value paradox with polyploidy, repeatomes, introns, phenomes and cell economy. Genes and Genomics 42, 699-714 (2020).

7. Nyonje, W. A. et al. Precision phenotyping and association between morphological traits and nutritional content in Vegetable Amaranth (Amaranthus spp.). J. Agric. Food Res. 5, 100165 (2021).

8. Sharma, S., Katoch, V., Kumar, S. \& Chatterjee, S. Functional relationship of vegetable colors and bioactive compounds: Implications in human health. J. Nutr. Biochem. 92, (2021).

9. Otunola, G. A. \& Martiryosan, D. Choosing suitable food vehicles for functional food products. Funct. Foods Heal. Dis. 11, 44-55 (2021).

10. Moghe, G. D., Leong, B. J., Hurney, S. M., Jones, A. D. \& Last, R. L. Evolutionary routes to biochemical innovation revealed by integrative analysis of a plant-defense related 
specialized metabolic pathway. Elife 6, (2017).

527 11. Vermeulen, R., Schymanski, E. L., Barabási, A.-L. \& Miller, G. W. The exposome and

health: Where chemistry meets biology. Science (80-. ). 367, 392-396 (2020).

12. FooDB. Available at: https://foodb.ca/. (Accessed: 25th June 2019)

13. Yannai, S. Dictionary of food compounds with CD-ROM. (Crc Press, 2012).

14. Kanehisa, M. \& Goto, S. KEGG: kyoto encyclopedia of genes and genomes. Nucleic Acids

Res. 28, 27-30 (2000).

15. Schläpfer, P. et al. Genome-wide prediction of metabolic enzymes, pathways, and gene clusters in plants. Plant Physiol. 173, 2041-2059 (2017).

16. Karp, P. D. et al. The BioCyc collection of microbial genomes and metabolic pathways.

Brief. Bioinform. 20, 1085-1093 (2019).

17. Soldatou, S. et al. Comparative Metabologenomics Analysis of Polar Actinomycetes. Mar.

Drugs 2021, Vol. 19, Page 103 19, 103 (2021).

18. Parkinson, E. I. et al. Discovery of the Tyrobetaine Natural Products and Their

Biosynthetic Gene Cluster via Metabologenomics. ACS Chem. Biol. 13, 1029-1037 (2018).

19. Saad, H. et al. Nocathioamides, Uncovered by a Tunable Metabologenomic Approach, 16479 (2021).

544 20. Fox Ramos, A. E., Evanno, L., Poupon, E., Champy, P. \& Beniddir, M. A. Natural products targeting strategies involving molecular networking: Different manners, one goal. Nat.

Prod. Rep. 36, 960-980 (2019).

547 21. Ranum, P., Peña-Rosas, J. P. \& Garcia-Casal, M. N. Global maize production, utilization, 
and consumption. Ann. N. Y. Acad. Sci. 1312, 105-112 (2014).

549

550

551

552

553

554

555

556

557

558

559

560

561

562

563

564

565

566

567

568

569

22. Milanlouei, S. et al. A systematic comprehensive longitudinal evaluation of dietary factors associated with acute myocardial infarction and fatal coronary heart disease. Nat. Commun. 11, 1-14 (2020).

23. Aharoni, A., Galili, G., Avni, A. \& Blazquez, M. Metabolic engineering of the plant primarysecondary metabolism interface This review comes from a themed issue on Plant biotechnology Edited. Curr. Opin. Biotechnol. 22, 239-244 (2011).

24. Murphy, K. M., Ma, L. T., Ding, Y., Schmelz, E. A. \& Zerbe, P. Functional characterization of two class ii diterpene synthases indicates additional specialized diterpenoid pathways in maize (zea mays). Front. Plant Sci. 871, 1-12 (2018).

25. Schmelz, E. A. et al. Biosynthesis, elicitation and roles of monocot terpenoid phytoalexins. Plant J. 79, 659-678 (2014).

26. Moore, V. M. \& Tracy, W. F. Combining ability of husk extension, maysin content, and corn earworm resistance. J. Am. Soc. Hortic. Sci. 146, 14-23 (2021).

27. Köllner, T. G., Degenhardt, J. \& Gershenzon, J. The product specificities of maize terpene synthases tps 4 and tps10 are determined both by active site amino acids and residues adjacent to the active site. Plants $\mathbf{9},(2020)$.

28. Schnee, C., Köllner, T. G., Gershenzon, J. \& Degenhardt, J. The maize gene terpene synthase 1 encodes a sesquiterpene synthase catalyzing the formation of (E)- $\beta$ farnesene, (E)-nerolidol, and (E,E)-farnesol after herbivore damage. Plant Physiol. 130, 2049-2060 (2002).

29. Chiorcea-Paquim, A. M., Enache, T. A., De Souza Gil, E. \& Oliveira-Brett, A. M. Natural 
phenolic antioxidants electrochemistry: Towards a new food science methodology.

Compr. Rev. Food Sci. Food Saf. 19, 1680-1726 (2020).

30. Kyraleou, M. et al. Discrimination of five Greek red grape varieties according to the anthocyanin and proanthocyanidin profiles of their skins and seeds. J. Food Compos.

Anal. 92, 103547 (2020).

31. Tang, K., Liu, T., Han, Y., Xu, Y. \& Li, J. M. The Importance of Monomeric Anthocyanins in the Definition of Wine Colour Properties. South African J. Enol. Vitic. 38, 1-10 (2017).

32. Dahab, M. A., Hegazy, M. M. \& Abbass, H. S. Hordatines as a Potential Inhibitor of COVID19 Main Protease and RNA Polymerase: An In-Silico Approach. Nat. Products Bioprospect. 10, 453-462 (2020).

33. Pihlava, J. M., Kurtelius, T. \& Hurme, T. Total hordatine content in different types of beers. J. Inst. Brew. 122, 212-217 (2016).

34. Totaro, G. et al. Elastomeric/antibacterial properties in novel random Ricinus communis based-copolyesters. Polym. Test. 90, 106719 (2020).

35. Scossa, F., Roda, F., Tohge, T., Georgiev, M. I. \& Fernie, A. R. The Hot and the Colorful: Understanding the Metabolism, Genetics and Evolution of Consumer Preferred Metabolic Traits in Pepper and Related Species. CRC. Crit. Rev. Plant Sci. 0, 1-43 (2019).

36. Sinisgalli, C. et al. The Beneficial Effects of Red Sun-Dried Capsicum annuum L. Cv Senise Extract with Antioxidant Properties in Experimental Obesity are Associated with Modulation of the Intestinal Microbiota. Mol. Nutr. Food Res. 65, 1-13 (2021).

37. de Oliveira, I. F. et al. Sorgoleone concentration influences mycorrhizal colonization in sorghum. Mycorrhiza 259-264 (2020). doi:10.1007/s00572-020-01006-1 
38. Schilmiller, A. L., Gilgallon, K., Ghosh, B., Jones, A. D. \& Last, R. L. Acylsugar acylhydrolases: Carboxylesterase-catalyzed hydrolysis of acylsugars in tomato trichomes. Plant Physiol. 170, 1331-1344 (2016).

39. Tikunov, Y. M., de Vos, R. C. H., Paramás, A. M. G., Hall, R. D. \& Bovy, A. G. A role for differential glycoconjugation in the emission of phenylpropanoid volatiles from tomato fruit discovered using a metabolic data fusion approach. Plant Physiol. 152, 55-70 (2010).

40. Covington, B. C., McLean, J. A. \& Bachmann, B. O. Comparative mass spectrometry-based metabolomics strategies for the investigation of microbial secondary metabolites.

Natural Product Reports 34, 6-24 (2017).

41. Trehan, S., Singh, N. \& Kaur, A. Diversity and relationship among grain, flour and starch characteristics of Indian Himalayan colored corn accessions. J. Food Sci. Technol. 57, 3801-3813 (2020).

42. Martínez-Cruz, M., Zenteno, E. \& Córdoba, F. Purification and characterization of a galactose-specific lectin from corn (Zea mays) coleoptyle. Biochim. Biophys. Acta - Gen. Subj. 1568, 37-44 (2001).

43. Belcaro, G. et al. Management of symptoms, pain and mobility with supplementary managements (including Movardol Forte) in osteoarthrosis: A 6-month, morphology supplement study. Minerva Ortop. e Traumatol. 71, 160-167 (2021).

44. Trammell, S. A. J. \& Brenner, C. Targeted, LCMS-based metabolomics for quantitative measurement of NAD+ metabolites. Computational and Structural Biotechnology Journal 4, e201301012 (2013).

45. Conze, D. B., Crespo-Barreto, J. \& Kruger, C. L. Safety assessment of nicotinamide 
riboside, a form of vitamin B 3. doi:10.1177/0960327115626254

615

616

617

618

619

620

621

622

623

624

625

626

627

628

629

630

631

632

633

634

635

46. Mehmel, M., Jovanovi'c, N. J. \& Spitz, U. Nicotinamide Riboside-The Current State of Research and Therapeutic Uses. doi:10.3390/nu12061616

47. Li, Y., Wang, Y. \& Wu, P. 5'-Methylthioadenosine and Cancer: old molecules, new understanding. J. Cancer 10, 927-936 (2019).

48. Shen, D. et al. Cell Death Discovery ADO/hypotaurine: a novel metabolic pathway contributing to glioblastoma development. Cell Death Discov. 7, 21 (2021).

49. Gao, P. et al. Hypotaurine evokes a malignant phenotype in glioma through aberrant hypoxic signaling. Oncotarget 7, 15200-15214 (2016).

50. Partyka, A., Rodak, O., Bajzert, J., Kochan, J. \& Nihanski, W. The Effect of L-Carnitine, Hypotaurine, and Taurine Supplementation on the Quality of Cryopreserved Chicken Semen. (2017). doi:10.1155/2017/7279341

51. Wang, C. et al. Pipecolic acid confers systemic immunity by regulating free radicals. Sci. Adv. 4, (2018).

52. Cheng, J., Chen, · Peng, Song, A., Wang, D. \& Wang, Q. METABOLIC ENGINEERING AND SYNTHETIC BIOLOGY-REVIEW Expanding lysine industry: industrial biomanufacturing of Iysine and its derivatives. J. Ind. Microbiol. Biotechnol. 45, 719-734 (2030).

53. Cheng, J. et al. An economically and environmentally acceptable synthesis of chiral drug intermediate I-pipecolic acid from biomass-derived lysine via artificially engineered microbes. J. Ind. Microbiol. Biotechnol. 45, 405-415 (2018).

54. Schulz, M. et al. Pantoea ananatis converts MBOA to 6-methoxy-4-nitrobenzoxazolin2(3H)-one (NMBOA) for cooperative degradation with its native root colonizing microbial 
consortium. Nat. Prod. Commun. 13, 1275-1278 (2018).

637

638

639

640

641

642

643

644

645

646

647

648

649

650

651

652

653

654

655

656

657

55. Fenz, R., Galensa, R. \& Ernst, L. Phenolcarbonsäuren und ihre Glycerinester in Maisgrits.

Zeitschrift für Leb. und Forsch. 19921943 194, 252-258 (1992).

56. Mahajan, N. et al. High fructose induced adipogenesis and inhibitory potential of trigonelline on murine mesenchymal stem cells: A morphological study. Int. J. Pharm. Sci.

Res. 10, 528-536 (2019).

57. Srinivasulu, C., Ramgopal, M., Ramanjaneyulu, G., Anuradha, C. M. \& Suresh Kumar, C. Syringic acid (SA) - A Review of Its Occurrence, Biosynthesis, Pharmacological and Industrial Importance. Biomed. Pharmacother. 108, 547-557 (2018).

58. Moreau, R. A., Nuñez, A. \& Singh, V. Diferuloylputrescine and p-coumaroylferuloylputrescine, abundant polyamine conjugates in lipid extracts of maize kernels. Lipids 36, 839-844 (2001).

59. Seaver, S. M. D. et al. The ModelSEED Biochemistry Database for the integration of metabolic annotations and the reconstruction, comparison and analysis of metabolic models for plants, fungi and microbes. Nucleic Acids Res. 49, D575-D588 (2021).

60. Wang, L., Xu, C., Qu, M. \& Zhang, J. Kernel amino acid composition and protein content of introgression lines from Zea mays ssp. mexicana into cultivated maize. doi:10.1016/j.jcs.2007.09.014

61. Li, K. et al. Large-scale metabolite quantitative trait locus analysis provides new insights for high-quality maize improvement. Plant J. 99, 216-230 (2019).

62. Opialla, T., Kempa, S. \& Pietzke, M. Towards a More Reliable Identification of Isomeric Metabolites Using Pattern Guided Retention Validation. Metabolites 10, 1-16 (2020). 
658

659

660

661

662

663

664

665

666

667

668

669

670

671

672

673

674

675

676

677

678

679

63. Claes, B. S. R., Takeo, E., Fukusaki, E., Shimma, S. \& Heeren, R. M. A. Imaging Isomers on a Biological Surface: A Review. Mass Spectrom. 8, (2019).

64. Kranenburg, R. F. et al. Mass-Spectrometry-Based Identification of Synthetic Drug Isomers Using Infrared Ion Spectroscopy. Anal. Chem. 92, 7282-7288 (2020).

65. Van Der Hooft, J. J. J. et al. Linking genomics and metabolomics to chart specialized metabolic diversity. Chem. Soc. Rev. 49, 3297-3314 (2020).

66. Huerta-Cepas, J., Serra, F. \& Bork, P. ETE 3: Reconstruction, Analysis, and Visualization of Phylogenomic Data. Mol. Biol. Evol. 33, 1635-1638 (2016).

67. Virtanen, P. et al. SciPy 1.0: fundamental algorithms for scientific computing in Python. Nat. Methods 17, 261-272 (2020).

68. Landrum, G. Rdkit: Open-source cheminformatics software. URL http://www. rdkit. org/, https://github. com/rdkit/rdkit (2016). Available at: https://www.rdkit.org/. (Accessed: 30th December 2020)

69. Patten, J. J. et al. Multidose evaluation of 6,710 drug repurposing library identifies potent SARS-CoV-2 infection inhibitors In Vitro and In Vivo. bioRxiv Prepr. Serv. Biol. 2021.04.20.440626 (2021). doi:10.1101/2021.04.20.440626 

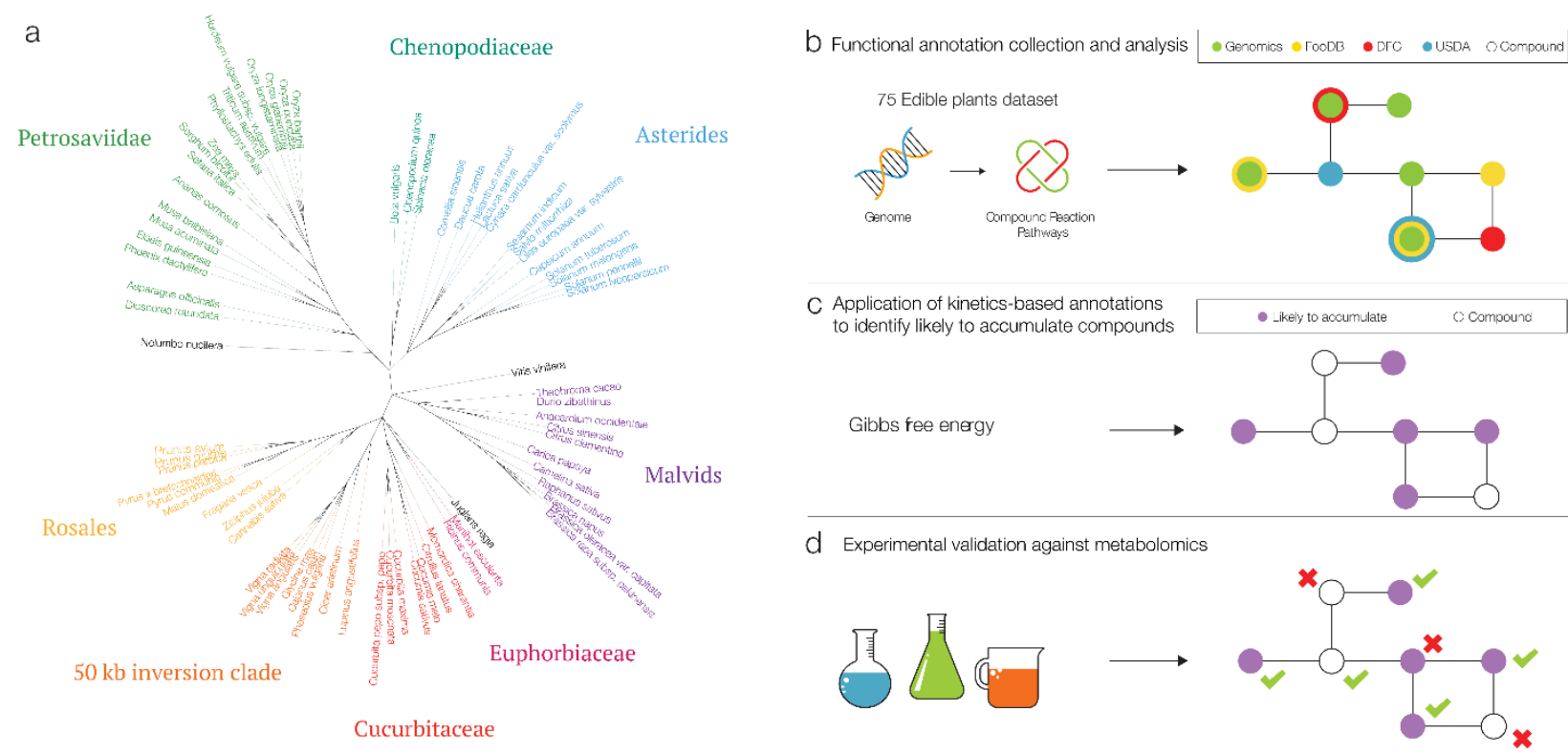

Figure 1 - Plant phylogenetic diversity and schematic overview of genomics contribution to

682 food composition knowledge. (a) Phylogenetic tree representing the 75 plants in our collection

683 colored by plant order. (b) Collection and evaluation of genomics-based annotations to food composition knowledge. Functional annotations include compounds, reactions and pathways. (c) Kinetics-based annotations help us to infer compounds likely to accumulate and hence experimentally detectable. (d) Validation of our kinetics-based approach against new metabolomics experiments, that detected compounds for 13 plants in our collection. 

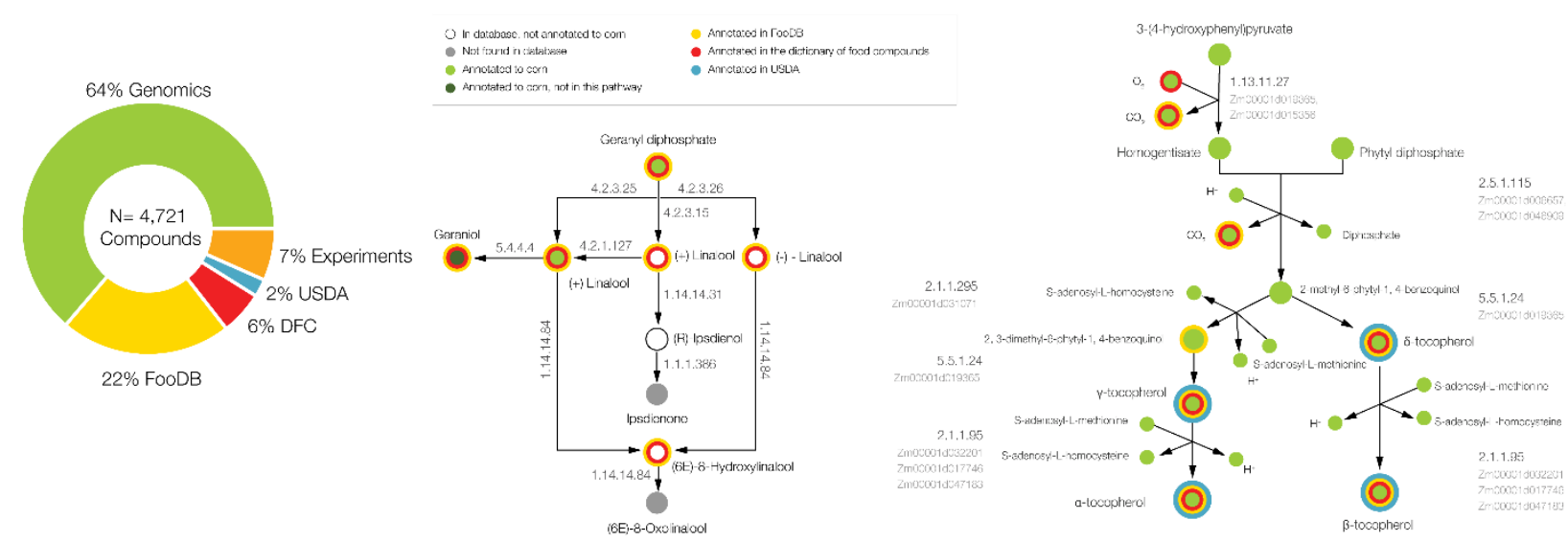

Figure 2 - Genomics-based annotations boost corn composition knowledge. (a) Database annotations for corn, indicating that some compounds are annotated in multiple databases. The total number $(\mathrm{N}=4,721)$ represents the number of unique compounds for corn after the addition of genomics-based annotations. Genomics databases are represented by KEGG and PlantCyc. Other food related databases used in this work are DFC (the dictionary of food compounds), FooDB and USDA. Finally, experiments denote the set of compounds collected by metabolomics experiments reported here for corn. (b) A partial adaptation of the Monoterpenoid biosynthesis pathway in corn (KEGG) showing annotation availability, overlap and gaps in the coverage of different databases and genomics-based annotations. The different colors denote annotation denote annotation sources as single or multiple concentric circles. 


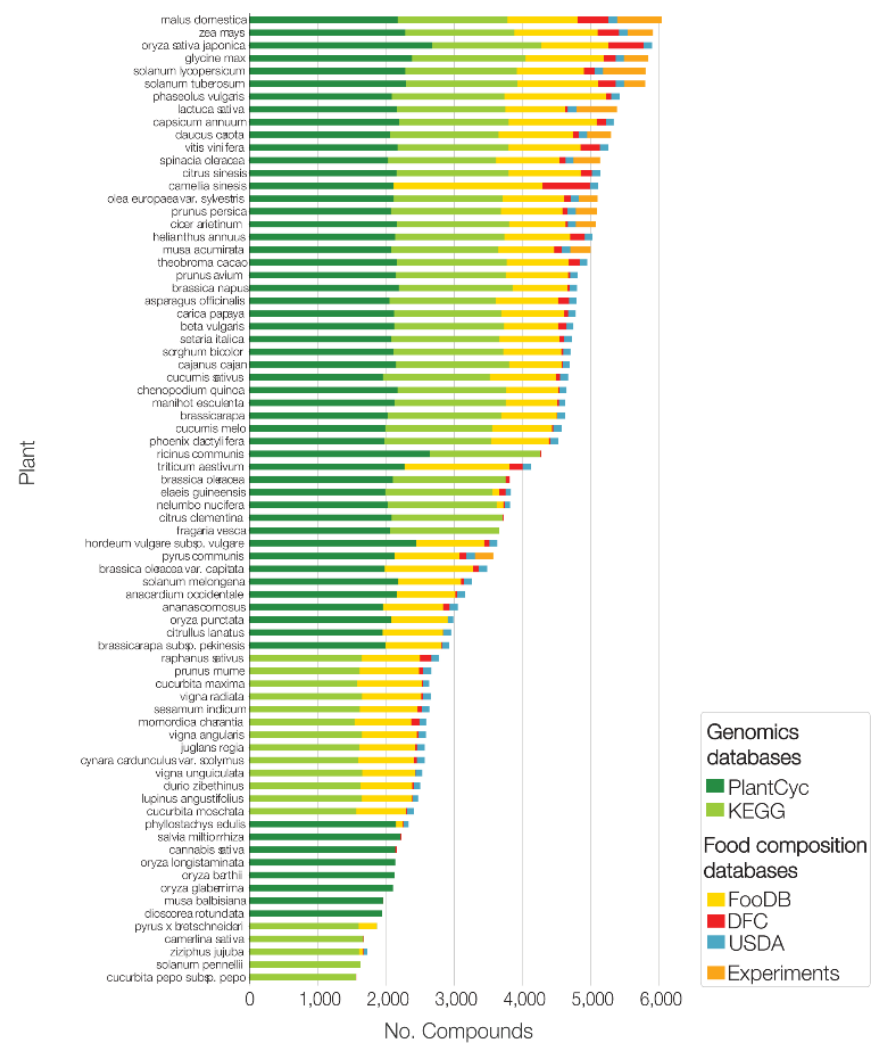

Figure 3 - Contribution of the different sources of food composition across the entire edible plant catalogue. Genomicsbased annotation are presented in two shades of green and include the KEGG (light green) and PlantCyc (dark green). Food composition databases include FooDB (yellow), DFC (red), USDA (blue). Compounds detected in metabolomics experiments are shown in orange. 
a
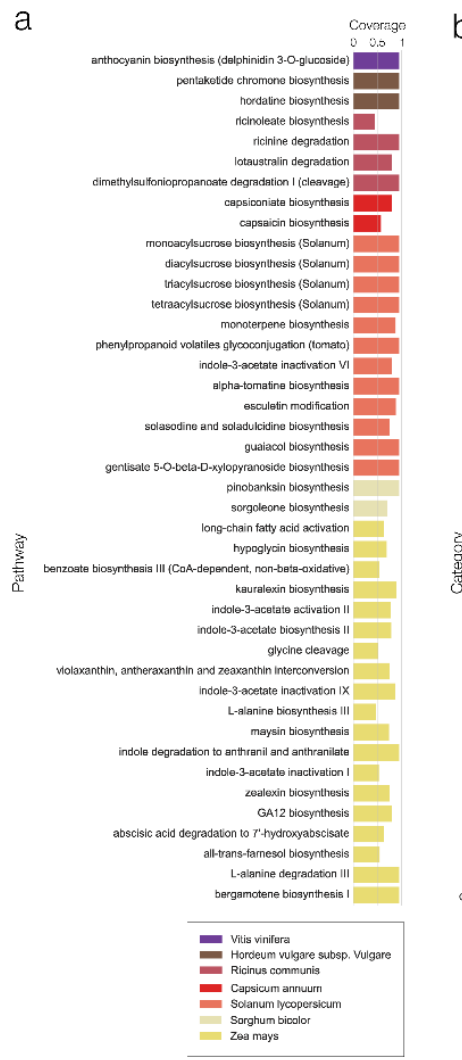

b

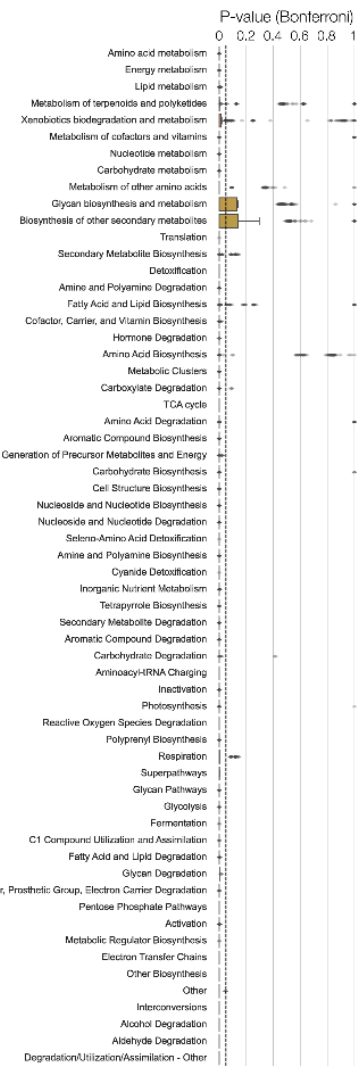

C

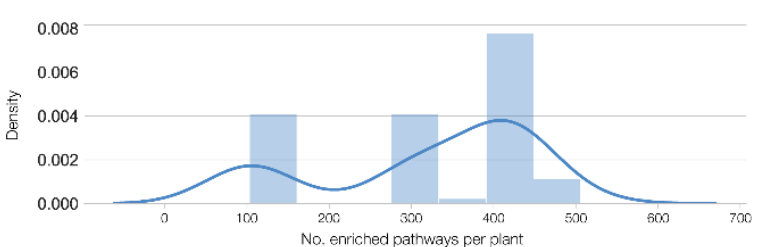

d

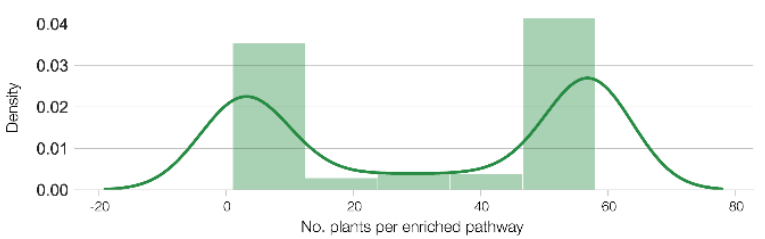

e

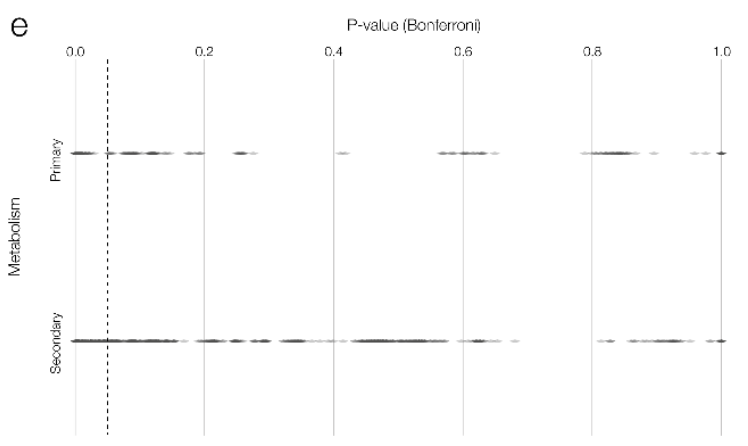

Figure 4 - Pathway enrichment analysis. (a) Plant specific significantly enriched pathways (hypergeometric test, $p<0.05$, Bonferroni multiple testing correction). The coverage of each pathway is defined by the ratio of present compounds to the total number of compounds in the reference pathway. These include signature known pathways like Capsaicin biosynthesis in pepper (red bars), acyl sugars and alpha tomatine pathways in tomato (light red bars) and maysin and zealexin biosynthesis (yellow bars). (b) A boxplot of the p-values (hypergeometric test, $p<0.05$, Bonferroni multiple testing correction) of pathway categories in corn. Medians found below the dashed line (0.05 enrichment line) represent the enriched pathways. We find that most pathways are enriched, indicating the diversity in our pathway coverage. (c) A density plot describing the distribution of enriched pathways per plant. We observed two peaks compatible with the two genomics databases included, KEGG (lower number of maps, each containing a larger number of reactions) and PlantCyc (larger number of maps, each containing a smaller number of reactions) (d) A density plot describing the distribution of the number of plants per enriched pathway. While most pathways are found in many plants, we observe several plant specific pathways described in detail in (a) and Figure S3. (e) A boxplot of enriched pathways (hypergeometric test, $\mathrm{p}<0.05$, Bonferroni multiple testing correction) classified as primary and secondary metabolism. Medians found below the dashed line (0.05 enrichment line) represent an enriched class. 

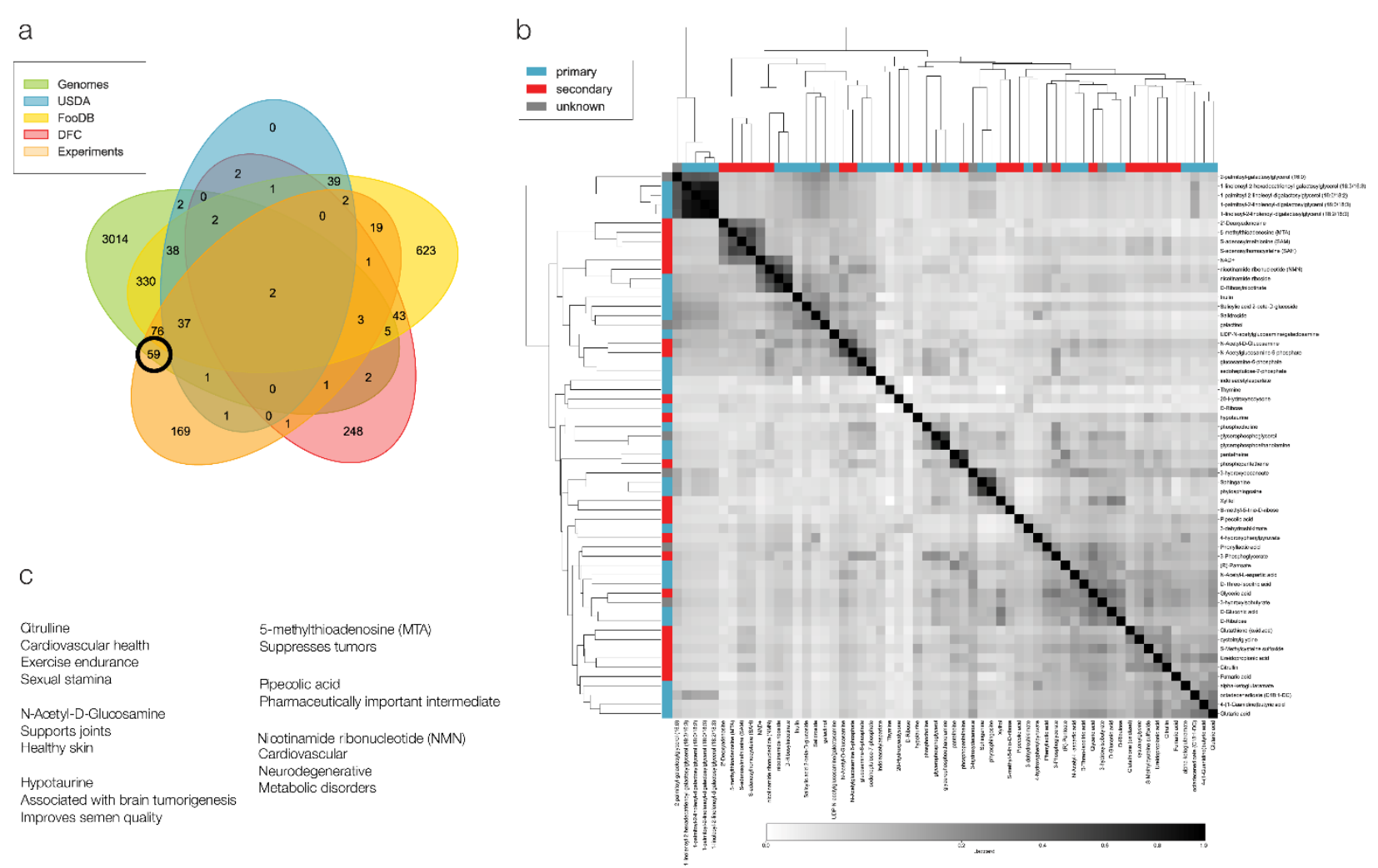

Figure 5 -Bioactive compounds in corn unveiled by genomics. (a) Venn diagram comparing all sources of data contributing to the corn compound collection. The black circle marks the fraction of compounds unique to genomics-based annotations and metabolomics experiments reported here. (b) A structural similarity based clustermap of the 59 compounds highlighted by the black

722 circle in panel (a). Compounds are classified to primary (blue) and secondary (red) metabolism

723 according to the pathways they are part of. Greyscale denotes structural similarity as Jaccard 724 distance, 0 being not similar and 1 being composed of the same bits in their vector representation. (c) The potential of corn as a nutritional influencer on wellbeing as learned with the addition of genome based-annotations, associated with antioxidant and anti-inflammatory 727 activity promoting, heart, skin and metabolic health. 
a

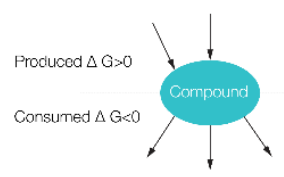

C

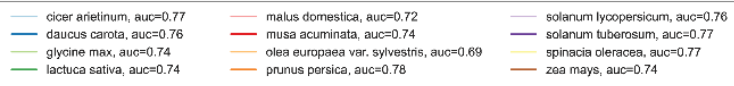

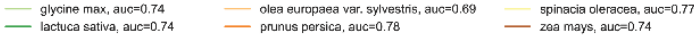

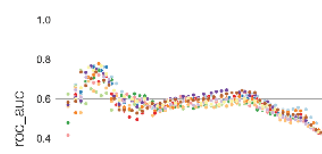

D.2

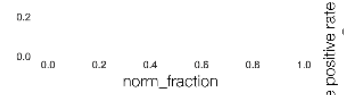

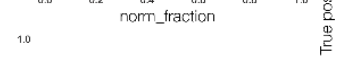

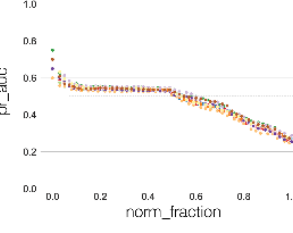

1.0

. score $=\sum \Delta G<0 \rightarrow$ consumed or score $=\sum \Delta G>0 \rightarrow$ accumulated

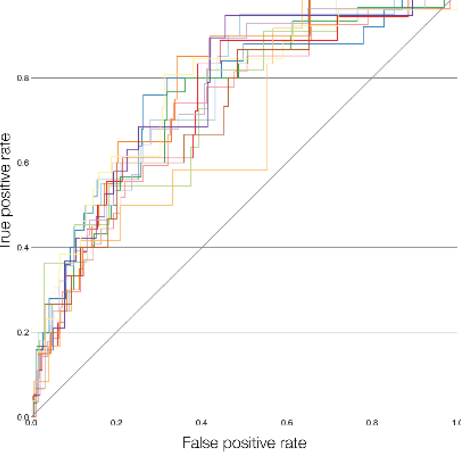

Vitamin E biosynthesis (tocopherols)

$\square$ Likely to be consumed - Likely to accurnulate Experimentaly detected - Unscored

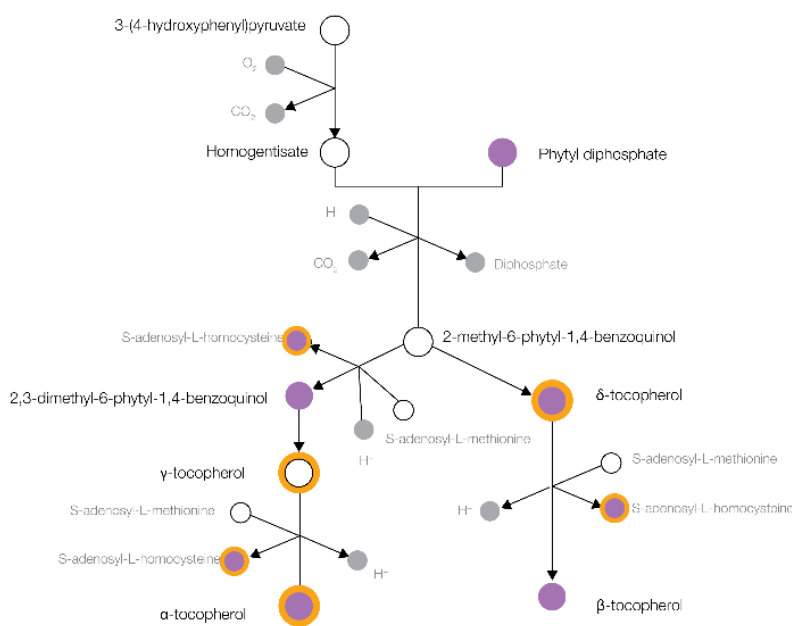

Figure 6 - Application of kinetics-based annotations to predict likely to accumulate compounds. (a)

Schematic description of the kinetics-based annotations approach. Gibbs free energy values, $\Delta G$, were collected for each reaction in each plant and used to calculate the cumulative score of a compound. If a compound is a reactant in a reaction, it gets a negative $\Delta G$ value and if it is a product, it gets a positive $\Delta G$ value. (b) Vitamin E biosynthesis (tocopherols) pathway of corn (PlantCyc), describing the possible outcomes of our approach. Circles are compounds and edges are reactions. (c) Performance of our approach including (i) optimal threshold analysis on the top-ranking compounds predicted to accumulate, establishing the window of best performance denoted by the area under the receiver operator curve, $A U C_{R O C}$ and the area under the precision recall curve, $A U C_{P R}$ for each plant, and (ii) the optimal receiver operating curves for plants included in this analysis. 